\title{
Proximity coefficients as a measure of interrelationships in sequences of behavior
}

\author{
PAUL J. TAYLOR \\ University of Liverpool, Liverpool, England
}

\begin{abstract}
Although a range of methods allow investigators to measure the local dependencies among behaviors in a sequence, only indirect methods are available for measuring the interrelationships among behaviors across an entire sequence. This article introduces a new "proximity" coefficient that measures interrelationships among behaviors as a direct function of their intrinsic organization within a sequence. The coefficient does not depend on a user-defined "window" of analysis and provides an efficient use of data that facilitates comparisons across actors, over time periods, and between single cases. An analysis of artificial data shows further properties of the coefficient, including a diagonal value that reflects the degree to which a behavior is reciprocated, and an asymmetry in values that depicts the relative precedence among behaviors. Extensions of the coefficient to the multivariate case, and its relation to existing methods of analyzing sequences, are discussed.
\end{abstract}

Several recent articles in this journal have presented new ways of extracting patterns of relations from sequences of behavioral events. Each applauds the insights that have come from using existing sequence methods, but also makes a case for why these methods do not fully capture the richness of the patterns in behavior sequences. Griffin (2000), for example, shows how exploring multisubject interaction as transitions over a single stream of behaviors does not capture the way that individuals jointly contribute to or coordinate the interaction process. Similarly, Magnusson (2000) provides evidence of patterns that often remain hidden because they are either distorted by intermittent events or located farther apart in the sequence than current methods of analysis consider. This last notion, that important associations lie beyond the stochastic "window" in which existing techniques measure relations, is the focus of this article. The article will review some limitations of existing approaches to analyzing sequences, and will argue that a notion of proximity captures the existing methodological viewpoints in a more flexible way. The proximity between behaviors in a sequence will be shown to be measurable by a new statistical coefficient, which does not depend on a user-defined "window" of analysis and provides an efficient use of data that facilitates comparisons across actors, over time periods, and between single cases.

\section{The Analysis of Behavioral Sequences}

The analysis considered in this article begins with an observed sequence of behavioral events, such as the dialogue

The author thanks Mehdi Mobli for his help in deriving the statistical equations for the $P$ coefficient. Correspondence should be addressed to P. J. Taylor, School of Psychology, Eleanor Rathbone Building, University of Liverpool, Liverpool L69 8PZ, England (e-mail: pjtaylor@ liverpool.ac.uk). of a hostage negotiation (Taylor, 2002a) or the events that lead up to a driving accident (Clarke, Forsyth, \& Wright, 1998). This sequence is parsed into a series of behavioral units (e.g., utterances or thought units) and these units are coded as one of a number of categories, which are usually predefined to capture conceptually meaningful variations in behavior over the sequence. The resulting sequence of behaviors may be examined for a variety of issues relating to the occurrence, co-occurrence, and ordering of behaviors over time. Investigators have traditionally taken one of two main approaches to measuring such relationships among the behaviors (Abbott, 1995).

Stochastic methods. One approach focuses on patterns and regularities in the step-by-step transitions among behaviors. The approach involves counting the number of times one behavior does and does not precede a second behavior, and from these counts computing a measure of the association between the two behaviors (Bakeman, McArthur, \& Quera, 1996; Fagen \& Mankovich, 1980; Wampold, 1989). In more sophisticated analyses, the frequencies may be combined with other conditional frequencies and submitted to a lag or log analysis as a way of determining the relative associations among combinations of behaviors (Chatfield \& Lemon, 1970; Iacobucci \& Wasserman, 1988). The resulting associations (or "chains" of these associations) may be interpreted directly to provide insights into the character and underlying units of different types of sequences (Gottman, Markman, \& Notarius, 1977; Taylor \& Donald, 2003). Alternatively, they may be compared across groups to determine whether or not the two groups differ with respect to the transitions of interest (Bakeman et al., 1996; Yoder, Bruce, \& Tapp, 2001).

By measuring the connections among a small number of adjacent behaviors, these stochastic methods excel in providing a detailed picture of the contingencies that shape a sequence. However, their restricted focus also makes it difficult to address more global questions about 
the organization of behavior, particularly in terms of understanding how the transitions come together to create the variety of frames (Drake \& Donohue, 1996), emphases (Taylor, 2002a), or paths (Holmes \& Sykes, 1993) that sequences take as they unfold over time. For example, an analysis of the major frames that individuals adopt in negotiation requires a method that captures larger scale groupings in the interrelationships among behaviors (Taylor, 2002a). Similarly, investigating the process by which groups make decisions requires an analysis that identifies how larger, coherent periods of behavior are ordered over time (Poole \& Roth, 1989). The problem highlighted by such examples is that important relationships lie beyond the limited window or focus of sequential association measures. These relationships might speak to the broader structure of the interaction process, as is the case when investigators identify phases or trends in behavior over time (Holmes \& Sykes, 1993; Taylor, 2002b). Or they might result from the delayed effect of certain behaviors on the sequence, as is the case when sequences contain a number of intermittent behaviors before a behavior reoccurs or is reciprocated (Magnusson, 2000; Putnam \& Jones, 1982). The importance of such relationships suggests that investigators would do well to increase the window or "zone of association" within which they measure the dependencies among behavioral events. It suggests that investigators should continue to focus on intrinsic associations, but that they should do so in a general way that encompasses the various degrees of association across a sequence.

Whole-sequence methods. The second approach to sequence analysis forgoes the detail offered by step-bystep approaches in an effort to capture the broader organization of behavior over the whole sequence (Abbott, 1995). One common method involves dividing a sequence into a series of smaller sub-sequences, wherein the cooccurrence of two behaviors may be measured using an association coefficient (Donohue, 1991; Taylor, 2002a). The resulting association value indicates the extent to which the two behaviors typically co-occur in the sequence, but it does so over a series of intervals large enough to capture the relative interrelationships among many different behaviors. The changing emphasis of such intervals is the focus of a second technique known as phase analysis. This analysis produces a "map" of the sequence that consists of a series of coherent phases, where each phase is an interval in which behavior has a single predominant substantive function (Holmes \& Sykes, 1993). As with other whole-sequence methods, the result of phase analysis is a macrolevel picture of the overall structure of the behavioral sequence.

These methods are excellent at identifying the major changes in behavior across a sequence. However, to derive this global picture, they typically measure the connections among behaviors indirectly, by imposing extrinsic criteria. For example, the analysis of behavioral relations within artificially created subsections of a sequence gives no consideration to the relationships among behaviors in different sections. Phase analysis moves even farther away from the data by grouping behaviors into a series of sub- sections that are imposed according to a set of external and somewhat arbitrary rules. As these examples suggest, whole-sequence methods achieve their representation of a sequence's structure indirectly, and not from the actual contingencies among behaviors. Although these methods can yield useful results, the relative value of their output will always depend on the tolerance of the data to the method's various assumptions. This suggests that investigators need a method whose results are comparable with those provided by existing whole-sequence techniques, but whose input consists of the intrinsic relations among cues and responses. Such a method, in line with the next stage for stochastic methods, requires a way of measuring interconnections among behaviors in a more general way.

\section{The Concept of Proximity}

I propose that the starting point for a general approach is the notion, inherent in current methods, of measuring (in one form or another) the relative collocation among behaviors in a sequence. This notion may be seen as a general proximity principle: Behaviors close together in a sequence have more in common -in terms of the actor's motivating concerns, strategies, and cognitions - than those far apart in the sequence. Proximity is basic to what allows our social interactions to make sense, with actions or utterances linking into other actions or utterances to shape a fluid, unfolding process. In face-to-face negotiation, for example, an individual is able to focus his or her dialogue on a chosen issue by using a particular group of behaviors, such as the repeated use of criticisms and insults to attack the other party's identity (Taylor, 2002a). These behaviors occur in close proximity and therefore have a quality in common that does not exist for behaviors that are not in close proximity.

The proximity principle assumes that previous behaviors in a sequence have an association with the current behavior, and that the extent of this association decreases with increasing temporal distance from the current behavior. This approach incorporates the stochastic nature of sequences by conceptualizing behavior as being more dependent on recently occurring behaviors than on behaviors that occurred farther in the past. It also retains the notion of behaviors holding relationships with all other behaviors in a sequence, because proximity is conceptualized as decreasing across the entire sequence. Thus, in conceptualizing behavior relationships by their relative collocation, the proximity principle extends the "zone of association" from a restrictive window of the sequence to the whole sequence. It provides a way of understanding the interrelationships among behaviors as per wholesequence methods, but it does this in the language of local cue-response relationships, as per stochastic methods. Proximity, therefore, provides a window into the world of global dynamics by looking at the local cue-response sequences.

Note that proximity is not a new concept to either theory or method. In the social interaction literature, proximity underlies the notion of limitation or channeling, whereby every exchange of messages is seen as narrowing down 
the probability that other categories of talk will occur (Putnam, 1985; Watzlawick, Beavin, \& Jackson, 1968). Those behaviors in immediate proximity may be shown to have the most influence on the direction of interaction development, but those farther back in history also have their own unique association with current behavior (Taylor \& Donald, 2003). The connection between proximity and interaction research also extends to theories that incorporate dimensions (Collins, 1981; Donohue, 1998), since these theories rest on the notion that behaviors close together in a sequence hold similar positions over the dimensions. To not do so would mean that the dimensions failed to capture behavioral dynamics in a coherent and meaningful way.

Proximity may also be seen in previous approaches to analyzing sequences. For example, when investigators divide a sequence into a series of sub-sequences and count frequencies of behaviors, they are imposing an artificial boundary whereby all those behaviors within the boundary are treated as having proximity and all those outside the boundary as not having proximity. Phase analysis examines proximity through a similar, stricter criterion that considers only uninterrupted sequences of identical behaviors as being related. Interestingly, the relationship between proximity and the degree of association between two behaviors is the focus of other analyses (e.g., Markov or log-linear analysis), which measure the extent to which one behavior can be used to predict future behavior. These methods consider directly the affect of proximal behaviors on the development of a sequence.

\section{COEFFICIENT OF PROXIMITY}

The evidence above suggests that the proximity concept may provide a powerful way of capturing the general interrelationships among behaviors in a sequence. To empirically measure proximity, an index is required that meaningfully captures the relative proximity among pairs of behaviors in a sequence. This index is proposed in the form of a proximity coefficient that expresses the interrelationships among types of behavior as a direct function of their relative placements in a sequence. A sequence is defined as a single stream of observations (e.g., ABABABCCD) in which different behaviors (e.g., A, B, $\mathrm{C}$, and D) may occur rarely or frequently, and in any order. As with many coefficients of association, the coefficient of proximity is constructed to vary between 0.00 and 1.00 . A zero coefficient for two behaviors, A and B, implies that the distance between the occurrence of $\mathrm{A}$ and $\mathrm{B}$ is maximum (i.e., the length of the sequence). A coefficient with a value of 1.00 implies that behavior A immediately precedes B throughout the sequence, with no exception. Coefficients with values between these two extremes reflect intermediate degrees of proximity between A and B. Specifically, values of the coefficient are calculated as a function of the number of acts separating relevant pairs of behaviors across the sequence. The exact nature of the function may differ depending on theoretical considerations, but a simple count of intervening behaviors suffices, in most cases. Regardless of the function used, the proximity coefficient decreases monotonically as more behaviors are found, on average, to separate the two behaviors being examined.

\section{Calculation of the Proximity Coefficient}

Consider the case of a single sequence of behaviors obtained from a coded social interaction. Let the behaviors in the sequence be denoted by $s_{i}(i=1,2, \ldots, n)$, where $i=1$ for the first behavior in the sequence and $i=n$ for the final behavior in the sequence. Thus, the sequence "EAB" would be indexed as $\mathrm{E}=s_{1}, \mathrm{~A}=s_{2}, \mathrm{~B}=s_{3}$, and $n=3$. The set of behaviors used to code the sequence will be denoted by an unordered set $V=\left\{v_{1}, v_{2}, \ldots, v_{m}\right\}$, where $m$ varies, depending on the number of coding categories used to represent behavior in the sequence. If $s_{i}$ is a specific occurrence of $v_{p}$ (where $p$ can be $1,2, \ldots, m$ ), then we write $s_{i}=v_{p}$. Finally, $n_{p}$ denotes the number of times a particular behavior occurs in the sequence.

The proximity coefficient between any two codes, $v_{p}$ and $v_{q}$, may be regarded as asking, for each $v_{p}$, to what extent one must move through the sequence to observe $v_{q}$. It is important to note that this question seeks to find the distance between a given $v_{p}$ and the first following instance of $v_{q}$, rather than every following instance of $v_{q}$. The search for the minimum distance between codes is both statistically and theoretically important. The search for minimum distance is statistically important for ensuring that the coefficient's value is not dependent on the number of times a given code appears within the sequence. If the coefficient was derived from a behavior's proximity to all following behaviors, there would be a tendency for frequently occurring codes to be associated with higher coefficients, since their greater occurrence would lead them to be positioned closer together in the sequence, on average, than were less frequent codes. The search for minimum distance is theoretically important for ensuring that proximity is measured constantly for relations across a sequence. For example, if there are only two occurrences of $A$ in a sequence, and both are immediately followed by $\mathrm{B}$, then the coefficient for A to B should specify maximum proximity regardless of whether the occurrences of $\mathrm{A}$ are positioned adjacent to one another or at opposite ends of the sequence. Of course, it remains possible that the second instance of B depends on both occurrences of $\mathrm{A}$, but the coefficient views such subsequent relations as being adequately captured by the relative differences among the behaviors' most immediate proximities. In cases in which it is known that the organization of a sequence hinges on successive dependencies, the coefficient might best be applied with more caution.

To find the first instance of $v_{q}$ from $v_{p}$, the proximity coefficient identifies the minimum difference in the indices associated with $v_{p}$ and subsequent instances of $v_{q}$ through

$$
\begin{aligned}
d\left(s_{i}=v_{p}, v_{q}\right)= & \min [j-i]-1 ; \\
& \text { for all } s_{j}=v_{q}, j>i,
\end{aligned}
$$

where $d\left(s_{i}=v_{p}, v_{q}\right)$ is the distance between an occurrence of code $v_{p}$ at position $i$ and the first occurrence of code 
$v_{q}$ at a position $j$ greater than $i$. The subtraction of 1 is necessary to ensure that $d\left(s_{i}=v_{p}, v_{q}\right)$ equals 0 when $v_{q}$ immediately follows $v_{p}$, since this is conceptualized as a case of perfect proximity (i.e., there are no intermediate behaviors).

Since $v_{p}$ may occur many times within a sequence, it is possible to obtain a better expression of $d\left(s_{i}=v_{p}, v_{q}\right)$ by averaging across every occurrence of $v_{p}$,

$$
\frac{1}{n_{p}} \sum_{s_{i}=v_{p}} d\left(s_{i}=v_{p}, v_{q}\right) \text {. }
$$

The proximity coefficient $(P)$ simply restates Equation 2 in a standardized form, as a proportion of $n$ :

$$
P\left(v_{p}, v_{q}\right)=1-\left(\frac{\sum_{s_{i}=v_{p}} d\left(s_{i}=v_{p}, v_{q}\right)}{n_{p}(n-2)}\right) .
$$

The addition to the denominator is $n-2$ rather than $n$ to give a count of the number of possible distances between the two end codes rather than simply the number of codes.

An inspection of the limits of $d\left(s_{i}=v_{p}, v_{q}\right)$ confirms that $P$ is bound between 0.00 and 1.00. Take the case of minimum proximity, in which $v_{p}$ occurs once at the beginning of the sequence (i.e., $s_{i}=1$ ) and $v_{q}$ occurs once at the end of a sequence (i.e., $s_{j}=n$ ). Restating Equation 1 in sample terms gives

$$
d\left(s_{i}=v_{p}, v_{q}\right)=[n-1]-1
$$

which, used with Equation 3, gives

$$
P\left(v_{p}, v_{q}\right)=1-\left(\frac{[n-1]-1}{n_{p}(n-2)}\right) .
$$

Since $n_{p}=1.00$, the members of the loss function in parentheses equate to 1.00 and $P\left(v_{p}, v_{q}\right)=0.00$. The upper limit $P\left(v_{p}, v_{q}\right)=1.00$ occurs only when $v_{q}$ always immediately follows $s_{i}$, and is similarly established by replacing $j$ with $(i+1)$ in Equation 1. This results in $d\left(s_{i}=v_{p}, v_{q}\right)=$ 0.00 , and, consequently, the loss function equating to 0.00 and $P\left(v_{p}, v_{q}\right)=1.00$.

Note that the coefficient described above is an example of a general approach to measuring the interrelations among behaviors of a sequence. One simple generalization of the equations given above is to introduce a weighting $w$ to the value returned by the numerator in Equation 2,

$$
\frac{1}{n_{p}} \sum_{s_{i}=n_{p}} w \times d\left(s_{i}=v_{p}, v_{q}\right) .
$$

The choice of weighting implicit in Equations 2 and 3 (i.e., $w=1$ ) measures proximity among behaviors independent of further theoretical restrictions. However, it is easy to perceive a situation in which it is appropriate to restrict the scope of proximity on the basis of theoretical or data considerations. For example, if the cue-responsecue-response sequence (i.e., triple-interact; see Taylor \&
Donald, 2003) is accepted as the "window" within which a particular cue influences the progress of interaction, then adopting $w=(n-2) / 4$, where $d\left(s_{i}=v_{p}, v_{q}\right)<(n-2)$ will limit estimation of proximity to only those behaviors found within the triple-interact window. In this situation, those behaviors outside of the four-behavior window are considered to have no proximity to those within the window and so add nothing to the resulting coefficient scores. Other choices do not necessarily involve arithmetic changes to the way distances among behaviors are measured. They may instead propose conditional weightings that return a particular value based on some property (quality) of the distance between $s_{i}$ and $s_{j}$ or some other external criterion.

One particularly important use of weighting occurs when investigators want their coefficients to measure the absolute distance between two codes. This will occur when an investigator wants to test hypotheses about specific differences in the proximity of two behaviors across different sequences. In the form presented above, $P$ expresses the distance between two codes as a proportion of the number of codes in the sequence. This makes the derived coefficient dependent on the length of the sequence examined. For example, if behavior A occurs only once at the beginning of a sequence, and behavior B occurs only once at the end of that sequence, $P_{(\mathrm{A}, \mathrm{B})}=0.00$ regardless of whether the sequence is $10 \mathrm{be}-$ haviors or 1,000 behaviors in length. However, behaviors $\mathrm{A}$ and $\mathrm{B}$ are separated by far more intervening events in a 1,000 -behavior sequence than in a 10 -behavior sequence, and investigators may want to capture this kind of disparity when conducting their comparisons.

To capture the absolute distance between behaviors, it is necessary to weight $d\left(s_{i}=v_{p}, v_{q}\right)$ by a ratio of the lengths of the sequences being compared. This ratio may be expressed in a general form as

$$
w=\frac{\left(n_{k}-2\right)}{\left(n_{k_{\max }}-2\right)},
$$

where $n_{k}$ is the number of codes that appear in the sequence for which the proximity coefficients are being calculated, and $n_{k_{\max }}$ is the number of codes that appear in the largest sequence of the data set. The weighting in Equation 7 simply rescores the distances observed between $v_{p}$ and $v_{q}$ in smaller sequences, as if they were distances observed in a sequence with length $n_{k_{\max }}$. The result is a loss function that is equivalent over different sequence lengths, thereby producing a set of proximity coefficients that reflect the absolute distance among codes and whose values may be directly compared.

\section{ILLUSTRATIVE EXAMPLE}

To illustrate the proximity coefficient in sample data, suppose we observe two interaction sequences. The left panel of Table 1 illustrates these sequences using letters to denote the occurrence of behaviors, with different letters indicating the occurrence of different types of behaviors. Sequence 1 involves five behaviors that occur in a 
Table 1

An Example of Two Behavior Sequences and

Their Resulting Proximity Coefficient Matrices

\begin{tabular}{|c|c|c|c|c|c|c|}
\hline \multirow[b]{3}{*}{ Event Sequence } & \multicolumn{6}{|c|}{ Resulting Proximity Coefficient Matrix } \\
\hline & \multirow{2}{*}{$\begin{array}{l}\text { Observation } \\
\text { Type }\left(v_{a}\right)\end{array}$} & \multicolumn{5}{|c|}{ Observation Type $\left(v_{b}\right)$} \\
\hline & & A & $\mathrm{B}$ & $\mathrm{C}$ & $\mathrm{D}$ & $\mathrm{E}$ \\
\hline \multirow{6}{*}{$\begin{array}{l}\text { Sequence 1: } \\
\text { E A B A B A B C C D }\end{array}$} & $\mathrm{A}$ & 88 & 100 & 63 & 38 & - \\
\hline & B & 100 & 88 & 75 & 50 & - \\
\hline & $\mathrm{C}$ & - & - & 100 & 94 & - \\
\hline & $\mathrm{D}$ & - & - & - & - & - \\
\hline & $\mathrm{E}$ & 100 & 88 & 25 & 0 & - \\
\hline & & A & $\mathrm{B}$ & $\mathrm{C}$ & $\mathrm{D}$ & $\mathrm{E}$ \\
\hline \multirow{6}{*}{$\begin{array}{l}\text { Sequence 2: } \\
\text { E A B A B A B C C D. } \\
\text { E A B A B A B C C D }\end{array}$} & $\mathrm{A}$ & 90 & 100 & 83 & 72 & 67 \\
\hline & $\mathrm{B}$ & 96 & 90 & 89 & 78 & 72 \\
\hline & $\mathrm{C}$ & 86 & 81 & 85 & 97 & 92 \\
\hline & $\mathrm{D}$ & 94 & 89 & 61 & 50 & 100 \\
\hline & $\mathrm{E}$ & 100 & 94 & 67 & 56 & 50 \\
\hline & & A & $\mathrm{B}$ & $\mathrm{C}$ & $\mathrm{D}$ & $\mathrm{E}$ \\
\hline \multirow{5}{*}{$\begin{array}{l}\text { Sequence } 3 \text { with absolute proximities: } \\
\text { E A B A B A B C C D }\end{array}$} & $\mathrm{A}$ & 94 & 100 & 83 & 72 & -9 \\
\hline & B & 100 & 94 & 89 & 78 & -9 \\
\hline & $\mathrm{C}$ & -9 & -9 & 100 & 97 & -9 \\
\hline & $\mathrm{D}$ & -9 & -9 & -9 & -9 & -9 \\
\hline & $\mathrm{E}$ & 100 & 94 & 67 & 56 & -9 \\
\hline
\end{tabular}

Note-Decimal points omitted.

sequence of 10 units. Sequence 2 involves the same five behaviors but is 20 units in length. Sequence 3 is a reanalysis of Sequence 1 that uses a different weighting of the proximities among behaviors. The matrices on the right panel of Table 1 give the proximity coefficients associated with each sequence.

An inspection of Sequence 1 shows that behavior E and behavior D occur only once, and at opposing ends of the sequence, so that their proximity is the minimum possible. In contrast, behavior B always occurs directly after behavior A, so that the proximity of these behaviors is the maximum possible. Consistent with these two limits, the coefficient matrix reports a perfect association between $\mathrm{A}$ and $\mathrm{B}$ (1.00) and a complete nonassociation between $\mathrm{E}$ and D (0.00). All of the other relationships among the codes have intermediate values that are dependent on their distances apart in the sequence. For instance, there are descending values of the coefficient for behavior A's relation with $\mathrm{B}$ (1.00), $C$ (.63), and D (.38). Examining the sequence confirms that behavior $\mathrm{A}$ is closest, on average, to behavior $\mathrm{B}$; is slightly less close to the two occurrences of behavior $\mathrm{C}$; and is farthest away from the concluding behavior, D.

The undefined value of the coefficient measuring the relationship of $\mathrm{A}$ to $\mathrm{E}$ is appropriate for Sequence 1, because $\mathrm{E}$ never follows A. While missing coefficients are an inevitable consequence of short sequences, the interpretation of missing coefficients within longer sequences can provide an indication of the relative distribution of behavior within the sequence. A large number of missing values in a variable row (or column) indicates that most observations of the behavior occurred toward the end (or beginning) of the sequence, as is the case for behavior $\mathrm{C}$ in Sequence 1. At the extreme, a column of missing values indicates a behavior that occurs only at the beginning of a sequence (e.g., behavior E in Sequence 1), whereas a row of missing values indicates a behavior that occurs only at the last position in the sequence (e.g., behavior D in Sequence 1).

Note that the matrix for Sequence 1 also reports a coefficient for a behavior preceding itself over the course of the sequence. Proximity coefficients on the diagonal of a matrix are meaningful and provide a measure of the amount of reciprocity (Putnam \& Jones, 1982) associated with the relevant behavior. The coefficients actually provide a graded measure of reciprocation, in the sense that they quantify the number of intervening codes that occur on average before reciprocation, rather than simply the proportion of immediate reciprocation. Thus, coefficients on the diagonal may be used to test hypotheses about the nature and breadth of reciprocity, such as the possibility that individuals delay their reciprocation to allow for intermediate behaviors that confirm the initial statement (Putnam \& Jones, 1982). In general, predictions about reciprocity or any other relationship may be defined in terms of the proximity between two behaviors, where deviation from the predicted proximity may be measured for its statistical likelihood (Efron \& Tibshirani, 1986). If the deviation is small enough, then it might be possible to conclude that the observed degree of reciprocity is unlikely to have occurred by chance.

Sequence 2 contains the same behaviors as Sequence 1 but includes a repeat of the 10 behaviors in Sequence 1 . The extra length of Sequence 2 provides sufficient observations to allow the calculation of proximity coefficients for all pairs of behavior (which is not necessarily true of existing methods), thereby leaving no empty cells in the matrix. As before, relative proximity in the sequence is reflected in the coefficients, with, for example, descend- 
ing values occurring for the relation of behavior A with $\mathrm{B}$ (1.00), C (.83), D (.72), and E (.67). Because Sequence 2 only repeats the ordering of behaviors in Sequence 1, the organization of behaviors remains constant across the two sequences and, consequently, so does the rank ordering of the coefficient values. However, the absolute values of the coefficients are higher for Sequence 2 when compared with Sequence 1. This results from the fact that proximity is estimated in the context of the complete sequence. Because it is not necessary to search through more than $50 \%$ of Sequence 2 to find an occurrence of A preceding $\mathrm{B}, \mathrm{C}, \mathrm{D}$, or E, the coefficients for behavior A are all above .50 . Indeed, in the current sequence, this happens to be the case for each of the 5 behaviors.

A second difference between the matrix produced from Sequence 1 and the matrix produced from Sequence 2 is asymmetry in the coefficient values. For example, the coefficient for behavior A preceding B (i.e., 1.00) is no longer identical to the coefficient for behavior B preceding A (i.e., .96). In general, matrices of proximity coefficients will be asymmetrical, reflecting the possibility that one behavior precedes the second behavior on the majority of occasions. For example, in Sequence 2, behavior A occurs before $\mathrm{B}$ on six occasions, whereas behavior B occurs before A on five occasions. This leads to a small difference of .04 (i.e., $1.00-.96=.04$ ) between the coefficients, which reflects the slight bias toward behavior A occurring before behavior B. Larger differences between coefficients suggest a greater asymmetry in the ordering of two behaviors, with a missing coefficient indicating that all instances of a behavior precede all instances of the second behavior.

Finally, Sequence 3 is a reanalysis of Sequence 1, in which the coefficients were weighted to make them directly comparable to those derived from Sequence 2. Specifically, the coefficients for Sequence 3 were calculated by applying the weighting given in Equation 7 with $n_{k_{\max }}=$ 20 , which is the number of behaviors in the larger comparison sequence, Sequence 2. One consequence of this weighting is that the absolute proximities measured for Sequence 3 are markedly higher than the coefficients for Sequence 1 (see Table 1). This occurs because $P$ expresses the distances among behaviors as a proportion of overall sequence length, so that they become relatively smaller (and consequently, $P$ larger) in the context of the longer 20-behavior sequence (i.e., Sequence 2) than in the original 10-behavior sequence. A second consequence of the weighting is that distances among behaviors are measured identically for both sequences. In this example, because Sequence 2 simply repeats the 10 behaviors of Sequence 3 , the relative distances among the behaviors is equivalent in both sequences. This equivalence is reflected in Table 1 by the identical values of the coefficients. For example, there are descending coefficients of identical value for the relation of behavior E with A (1.00), B (.94), C (.67), and D (.56). As before, the shortness of Sequence 3 leads to the missing value associated with the relation $\mathrm{E}$ to itself, which is computable for Sequence 2. The shortness of Sequence 3 is also responsible for discrepancies in the values of the coefficients on the diagonal of the matrix.

\section{EXTENSIONS AND RELATIONSHIPS}

\section{Extensions in Analytical Techniques}

Multivariate proximities. An important property of $P$ is that it is analytical in $d$, facilitating multivariate extensions. The treatment above has been for the case of assigning each unit one behavioral code, but it is possible to compute a single coefficient for cases in which units are assigned codes from more than one coding scheme. One approach is to calculate conditional proximities of the form

$$
\begin{gathered}
P\left(v_{p_{1}}, v_{q_{1}}: v_{p_{2}}, v_{q_{2}}: \ldots: v_{p_{k}}, v_{q_{k}}\right) \\
=1-\frac{w_{1} \times d\left(s_{i}=v_{p_{1}}, v_{q_{1}}\right)}{n_{p_{1}}(N-2)} \\
\times \frac{w_{2} \times d\left(s_{i}=v_{p_{2}}, v_{q_{2}}\right)}{n_{p_{2}}(N-2)} \\
\times \cdots \times \frac{w_{k} \times d\left(s_{i}=v_{p_{k}}, v_{q_{k}}\right)}{n_{p_{k}}(N-2)},
\end{gathered}
$$

where each $d\left(s_{i}=v_{p_{k}}, v_{q_{k}}\right)$ is a different coding applied to the interaction sequence, $w_{k}$ is the weighting for that coding, and $n_{p_{k}}$ is the number of times the behavior from that coding occurs in the sequence. This approach produces a coefficient that denotes the proximity in which a particular set of behaviors (i.e., each $v_{q}$ ) follow the codes assigned to the current behavior (i.e., each $v_{p}$ ). The possibility of measuring the interrelationships among several behavioral streams should be useful, for example, to investigators who want to map the connections between individuals' verbal behavior and their self-reported "stream of thought" (Sillars, Roberts, Leonard, \& Dun, 2000). A set of associations might be predicted between the structure of cognitive units and groups of behaviors, with future analyses examining how changes in context or person variables mediate these connections. Other extensions might combine an analysis of dialogue with an analysis of nonverbal cues (Beattie \& Shovelton, 2002), or simultaneously examine substantive and relational aspects of dialogue by employing the relevant coding schemes (Donohue, 1998).

Testing the null hypothesis. In some circumstances, investigators may want to evaluate whether or not the proximity observed between two behaviors is likely to have occurred by chance. Specifically, they may want to evaluate whether or not an observed coefficient is important (e.g., high in value) by comparing it to the value expected for that coefficient if behaviors were distributed randomly in the sequence. For example, an investigator of parent-child interaction may want to evaluate whether the reciprocation of a parent's smile, as measured by $P_{\text {(Smile, Smile) }}$, is significantly higher than the $P_{\text {(Smile, Smile) }}$ expected under the null hypothesis of chance reciprocation. Since the observed $P$ coefficient will be interpreted differently depending on how much it deviates from the expected value, it is im- 
portant to have a method of estimating the distribution of $P$ under the null hypothesis. One solution to this problem is to permute the observed sequence many times (e.g., 10,000 times) while calculating $P$ coefficients for each permutation (Efron \& Tibshirani, 1986). This procedure would provide the empirical distribution of each $P\left(v_{p}, v_{q}\right)$ under the hypothesis of randomness, from which a $p$ value for the observed $P\left(v_{p}, v_{q}\right)$ may be estimated by locating its value in the empirical distribution. The nearer the observed $P\left(v_{p}, v_{q}\right)$ to the tails of the empirical distribution, the more confident an investigator can be that the proximity between $v_{p}$ and $v_{q}$ did not occur by chance.

Comparison of two coefficients. Some investigators may be keen to test the difference between two proximity coefficients or mean coefficient values. Comparisons of coefficient values may be used to test the relationship between a range of independent variables and the organization of speakers' cues and responses. For example, a comparison of coefficients derived from actual negotiations and training simulations provides a detailed account of the various differences in interaction dynamics between these two contexts. The typical behavioral chains, the impact of different strategies on the other party's response, and the long-term ordering of behavior are just three aspects of behavior that can be examined by comparing such matrices. For most purposes, such comparisons may be achieved by computing a permutation test on the differences among coefficient values, as outlined by Efron and Tibshirani (1986), Bakeman et al. (1996), and others. The analysis may assume independence of sampling when calculations are made across different sequences but would require adjustment to account for dependence among observations when comparing within a single sequence. Interestingly, two different possibilities are available for estimating the sampling distribution in this scenario. When comparing groups of sequences, the distribution may be derived through random sampling of the same coefficient in the different sequences. However, for small samples, it is also possible to estimate the extremity of a particular difference by deriving a distribution of differences from all possible pairings of coefficients in the proximity matrix. The permutation approach is therefore likely to provide the most appropriate test of differences in small and large data sets.

Extensions to time sequence data. The development of recording technologies has increasingly led investigators to record and analyze data in which the relative timing and duration of events are represented. This form of data, known as timed event sequence data (Bakeman \& Quera, 1995), may in principle be analyzed by proximity coefficients that use the difference between offset and onset times as the basis for measuring $d\left(s_{i}=v_{p}, v_{q}\right)$. Specifically, the modified coefficient would replace the index $i$ with a count of seconds elapsed from the beginning of the sequence, so that $d\left(s_{i}=v_{p}, v_{q}\right)$ reflects the gap in seconds between the offset of $v_{p}$ and the onset of $v_{q}$. $P$ would then reflect the gap between the offset and onset times as a proportion of the sequence's total length in seconds. When calculated in this way, $P$ coefficients on the off-diagonal of a matrix may be interpreted in a similar way to that described above, with lower values indicating lower proximity within the sequence. In contrast, coefficients on the diagonal of the matrix will have a different interpretation, which relates to the average duration of occurrence of the relevant behavior. The attractive feature of all these coefficients, however, is that they represent a general measure that does not depend on a specified time window or target event to measure the relationships among behaviors (Bakeman \& Quera, 1995).

\section{Relationships With Other Techniques}

To help round out the picture of where proximity coefficients fit as a technique for analyzing sequences, it may be useful to show that $P$ has precise relationships with other sequence methods.

Sequential association measures. As outlined by Bakeman et al. (1996), existing indices such as phi or kappa are successfully used to measure the strength of association or transitional likelihood among adjacent behaviors. These indices measure the specific case of immediate proximity, in which only neighboring behaviors are conceptualized as having joint influence on interaction. The indices are therefore equivalent to proximity coefficients that are weighted to disregard behaviors that do not immediately follow $v_{p}$. This equivalence is not perfect, however, since the weighted $P$ would be calculated from measures of how often a target behavior does and does not follow $v_{p}$, whereas most association indices also consider how often the absence of $v_{p}$ is and is not followed by the target behavior. The difference here is that most association indices equate joint absence of two behaviors as indicating a stronger association, whereas $P$ does not make this assumption and relies on a conditional likelihood as an estimate of the relationship between the behaviors. This offers an important theoretical alternative, particularly in cases in which the investigator is unsure whether the behavior did not occur or was not observed as occurring (Dice, 1945; Taylor, Bennell, \& Snook, 2002).

Lag sequence analyses. Lag analysis exemplifies a number of methods that examine the relative frequencies of immediate and longer term transitions among behaviors (Bakeman \& Quera, 1995). Conceptually, lag analysis looks at specific instances of proximity, with lag 1 relating to what appears in immediate proximity, lag 2 relating to what appears in less proximity, and so on. The analysis may be replicated by using a series of weightings, with the relevant proximity values for each weighting plotted in the same way investigators plot behavioral occurrence across different lags (Putnam \& Jones, 1982). However, this approach defeats the purpose of the proximity coefficient, which is to combine all of the lags into one coefficient that denotes the average occurrence of higher lags (i.e., further distance between behaviors) with a lower coefficient value.

Gamma analysis. Gamma analysis is a set of nonparametric statistics that provide a measure of the general order of behaviors in a sequence and a measure of the distinctiveness or overlap of behavior types (Pelz, 1985). Psychological research has typically used three measures: Pelz's gamma, which measures the proportion of A behaviors 
that precede or follow B behaviors in a sequence; precedence scores, which indicate the location of the behaviors in the overall ordering of element types; and separation scores, which give an indication of the relative distinctiveness of behavior types. There is no direct relation between the proximity coefficient and Pelz's gamma or precedence scores, because $P$ is based on distances between behaviors and not on the relative ordering of the elements. However, a parallel measure is given by the disparity in $P$ for any two behaviors (i.e., $\left.P_{(\mathrm{A}, \mathrm{B})}-P_{(\mathrm{B}, \mathrm{A})}\right)$, where the resulting value provides an indication of the difference in likelihood of A preceding or succeeding B. A larger disparity indicates a greater asymmetry in the ordering of occurrences among the two behavior types.

In contrast to the above indirect relationship, a direct relation exists between separation scores and the diagonal of a proximity coefficient matrix. Coefficients on the diagonal denote the extent to which a single behavior reciprocates without intervening or "separating" behaviors. A high coefficient on the diagonal will indicate a relatively coherent, separate period of occurrence for the relevant behavior. In contrast, a low coefficient suggests little separation of the behavior from other acts in the sequence. However, whereas a separation score measures the extent to which a behavior forms a single coherent sub-sequence, the value of the proximity coefficient is less stringent and allows for the possibility of two or more coherent sub-sequences occurring across an interaction. This should be particularly useful for investigators analyzing the structure of interactions that are likely to repeat the same phases of action (e.g., decision-making meetings, Poole \& Roth, 1989).

Phase analysis. Phase analysis represents the pattern of behaviors in a sequence by providing a serial map of coherent periods or phases of interaction (Holmes \& Sykes, 1993). The approach enacts a strict case of proximity, in which behaviors are considered related only when they are part of a sequence of identical behaviors or a sequence of identical behaviors with a predefined number of exceptions. Because the proximity coefficient is more flexible than this all-or-none criterion, it is able to provide a refined picture of interaction phases. Specifically, the reciprocation values of the proximity coefficient (e.g., $\left.P_{(\mathrm{A}, \mathrm{A})}\right)$ indicate the extent to which a particular type of phase occurs, with higher values reflecting greater occurrence of phases. The off-diagonals of a proximity coefficient matrix indicate the ordering of phases. For example, phase analysis is often used to test the prediction that interactions move through a number of coherent phases (Holmes \& Sykes, 1993; Poole \& Roth, 1989). Within the framework of proximity, a perfect sequence of phases would be represented by a (rearranged) matrix in which the upper off-diagonal coefficients were missing (since previous phases should not occur again), the coefficients on the diagonal equaled 1.00 (since phases are defined as uninterrupted occurrences of a particular code), and the lower off-diagonal coefficients were monotonically decreasing in a manner that represented the order of phases. Coefficient values of less than 1.00 on the diagonal would indicate a separation or recycling of the phases. A detailed analysis of the lower matrix coefficients would give some indication as to whether reoccurring phases had a common predecessor.

Optimal matching. An extension of phase analysis, known as optimal matching analysis, consists of techniques that compute either the overall similarity of two or more sequences or the similarity of these sequences to a prototypical sequence (Holmes, 1997; Sankoff \& Kruskal, 1983). The result of optimal matching is a dissimilarity score that can be compared with other scores to give an indication of the differences among sequences (e.g., through multidimensional scaling). Although proximity offers no direct comparison to this approach, it is possible to generate a similar analysis by comparing matrices of proximity coefficients computed from different sequences. Specifically, the disparity between two matrices may be used as a measure of the dissimilarity between the associated sequences, with greater disparity indicating a larger difference in the behavioral organization of each sequence. By computing a measure of dissimilarity for two matrices, and repeating this calculation for every pair of matrices derived from a data set, it is possible to generate a set of coefficients that measure sequence dissimilarities in a way comparable to optimal matching. A further, detailed comparison of the matrices also makes it possible to uncover the major variations in cue-response contingencies that underlie the similarities among sequences as a whole. Such an approach to sequence comparison has the advantage of not requiring the stipulation of substitution costs, which are external to the data and can dramatically affect the results of optimal matching analysis.

\section{Conclusions}

This article sought to define an empirical method of quantifying the interrelationships among sequences of behaviors. Most existing research has measured these relationships indirectly, either by imposing extrinsic divisions on the data (see, e.g., Taylor, 2002a) or by focusing on consistencies in immediate cue-response transitions (see, e.g., Bakeman \& Quera, 1995). However, to make fully empirical our conceptual theories about behavioral processes, it is beneficial to introduce a precise way of measuring the overall structure of localized connections among behaviors (Collins, 1981). The concept proposed in this article was proximity: The closer two behaviors occur in a sequence, the more they have in common conceptually. To measure proximity, a coefficient was introduced that expresses the interrelationships among behaviors as a direct function of their relative placements in a sequence. The coefficient is a general, computationally simple measure that avoids the arithmetic manipulations and extrinsic assumptions about the breadth of relations made by many existing techniques. It also boasts other properties, including a method of analyzing reciprocation and asymmetry among occurrences of behavior, and an efficient use of data that enables comparisons across speakers, among transcripts, and across different sections of the same sequence. Future work may therefore use the 
coefficient to investigate how complex cue-response sequences underlie the global patterns of movement that are observed over dimensions and constructs of social interaction. To aid investigators in this work, a Perl executable file that calculates the coefficient matrices for behavioral sequences has been developed. It is freely available from the author on request.

\section{REFERENCES}

Аввотт, A. (1995). Sequence analysis: New methods for old ideas. Annual Review of Sociology, 21, 93-113.

Bakeman, R., McArthur, D., \& Quera, V. (1996). Detecting group differences in sequential association using sampled permutations: Log odds, kappa, and phi compared. Behavior Research Methods, Instruments, \& Computers, 28, 446-457.

Bakeman, R., \& Quera, V. (1995). Analyzing interaction: Sequential analysis with SDIS and GSEQ. New York: Cambridge University Press.

Beattie, G., \& Shovelton, H. (2002). What properties of talk are associated with the generation of spontaneous iconic hand gestures? British Journal of Social Psychology, 41, 403-417.

Chatfield, C., \& Lemon, R. E. (1970). Analysing sequences of behavioural events. Journal of Theoretical Biology, 29, 427-445.

Clarke, D. D., Forsyth, R. S., \& Wright, R. L. (1998). Junction road accidents during cross-flow turns: A sequence analysis of police case files. Accident Analysis \& Prevention, 31, 31-43.

Collins, R. (1981). On the microfoundations of macrosociology. American Journal of Sociology, 86, 984-1014.

DicE, L. R. (1945). Measures of the amount of ecologic association between species. Ecology, 26, 297-302.

Donohue, W. A. (1991). Communication, marital dispute, and divorce mediation. Hillsdale, NJ: Erlbaum.

DonohuE, W. A. (1998). Managing equivocality and relational paradox in the Oslo peace negotiations. Journal of Language \& Social Psychology, 17, 72-96.

Drake, L. E., \& Donohue, W. A. (1996). Communicative framing theory in conflict resolution. Communication Research, 23, 297-322.

EFron, B., \& TiBShIRANi, R. (1986). Bootstrap methods for standard errors, confidence intervals, and other measures of statistical accuracy. Statistical Science, 1, 54-77.

FAgen, R. M., \& Mankovich, N. J. (1980). Two-act transitions, partitioned contingency tables, and the "significant cells" problem. Animal Behaviour, 28, 1017-1023.

Gottman, J., Markman, H., \& Notarius, C. (1977). The topography of marital conflict: A sequential analysis of verbal and nonverbal behavior. Journal of Marriage \& the Family, 39, 461-477.

GRIFFIN, W. A. (2000). A conceptual and graphical method for converging multisubject behavioral observational data into a single process indicator. Behavior Research Methods, Instruments, \& Computers, 32, 120-133.

HoLmEs, M. E. (1997). Optimal matching analysis of negotiation phase sequences in simulated and authentic hostage negotiations. Соттиnication Reports, 10, 1-8.

Holmes, M. E., \& Sykes, R. E. (1993). A test of the fit of Gulliver's phase model to hostage negotiations. Communication Studies, 44, 38-55.

IACOBuCCI, D., \& WasSERMAN, S. (1988). A general framework for the statistical analysis of sequential dyadic interaction data. Psychological Bulletin, 103, 379-390.

Magnusson, M. S. (2000). Discovering hidden time patterns in behavior: T-patterns and their detection. Behavior Research Methods, Instruments, \& Computers, 32, 93-110.

Pelz, D. C. (1985). Innovation complexity and the sequence of innovating stages. Knowledge: Creation, Diffusion, Utilization, 6, 261-291.

Poole, M. S., \& Roth, J. (1989). Decision development in small groups: IV. A typology of group decision paths. Human Communication Research, 15, 323-356.

Putnam, L. L. (1985). Bargaining as task and process: Multiple functions of interaction sequences. In R. L. Street, Jr. \& J. N. Cappella (Eds.), Sequence and pattern in communicative behaviour (pp. 225242). London: Edward Arnold.

Putnam, L. L., \& Jones, T. S. (1982). Reciprocity in negotiations: An analysis of bargaining interaction. Communication Monographs, 49, 171-191.

Sankoff, D., \& Kruskal, J. B. (1983). Time warps, string edits, and macromolecules: The theory and practice of sequence comparison. Reading, MA: Addison-Wesley.

Sillars, A., Roberts, L. J., Leonard, K. E., \& Dun, T. (2000). Cognition during marital conflict: The relationship of thought and talk. Journal of Social \& Personal Relationships, 17, 479-502.

TAYLOR, P. J. (2002a). A cylindrical model of communication behavior in crisis negotiations. Human Communication Research, 28, 7-48.

TAYLOR, P. J. (2002b). A partial order scalogram analysis of communication behavior in crisis negotiation with the prediction of outcome. International Journal of Conflict Management, 13, 4-37.

TAYlor, P. J., Bennell, C., \& SNOOK, B. (2002). Problems of classification in investigative psychology. In K. Jajuga, A. Sokolowski, \& H.-H. Bock (Eds.), Classification, clustering, and data analysis: Recent advances and applications (pp. 479-487). Heidelberg: Springer.

TAYLOR, P. J., \& DonalD, I. (2003). Foundations and evidence for an interaction-based approach to conflict negotiation. International Journal of Conflict Management, 14, 213-232.

WAMPOLD, B. E. (1989). Kappa as a measure of pattern in sequential data. Quality \& Quantity, 23, 171-187.

Watzlawick, P., Beavin, J. H., \& Jackson, D. D. (1968). Pragmatics of human communication: A study of interactional patterns, pathologies, and paradoxes. London: Faber.

Yoder, P. J., BRUce, P., \& TAPP, J. (2001). Comparing sequential associations within a single dyad. Behavior Research Methods, Instruments, \& Computers, 33, 331-338.

(Manuscript received August 6, 2004; revision accepted for publication January 21,2005 .) 